\title{
165. Studies on Head Injury: On the Cerebral Hemorrhage in the Area of Occipital Lobe
}

\author{
Masatoshi Honma and Kiyoshi Sato \\ 2nd Department of Surgery, Juntendo University, School of Medicine \\ Michio INUI \\ Tokyo Metropolitan Medical Examiner Office
}

Cerebral circulatory disturbance on the acute head injury takes place of some questions about it's pathogenesis. Recently we carried out 3 autopsy cases of hemorrhagic infraction which localized in occipital regions.

This report is one of these cases, which shows interesting matters in genesis of bleeding.

Case: Miss H.S. aged 43, on 26 November 1966, she fell down from stairs and stroke on the occipital region, her consciousness was clear, when she complained of slight headache, anorexia, nausea and vomitting two days after the injury. On 2 December 1966, consciousness disappeared suddenly, and one hour later she died.

Autopsy findings show: 1) localized cerebral purpura of occipital and temporal lobes with diffuse petechial hemorrhage in cortex and subcortical white matter. 2) slightly organized thrombosis of lateral sinus and inferior cerebral vein, lefts. 3) subcutaneous hemorrhage of occipital and frontal regions. 4) gastric perforation of acute ulcer due to head injury. Microscopically, the cortex and subcortical white matter of occipital and temporal lobes showed ring hemorrhage of capillares, arterioles and venous congestion.

In this case, the organizing thrombosis in left lateral sinus and inferior cerebral vein is a main role of this circulatory disturbance. These cerebral purpura was happened by destraction of arteriole in this circulatory area of inferior cerebral vein , and cortical and subcortical arteries.

\section{Studies pertaining to the Airborne Landing Fall. Report No. 7. (Special Clinical Cases.)}

Seiji Hosoda, Hironobu Shimada, Satoru Watanabe and Hirota Horinouchi

Department of Neurosurgery, Self-Defense Forces Central Hospital

The etiology of the disorders sustained by the airborne landing fall training is due to the landing shock and parachute opening shock, as previously reported in 